\title{
Enhancing Interactivity with Transcranial Direct Current Stimulation
}

\section{Bo Wan}

School of Computer Science,

Xidian University

wanbo@xidian.edu.cn

Chi Vi

SCHI lab, University of Sussex

C.Vi@sussex.ac.uk

\section{Abstract}

Transcranial Direct Current Stimulation (tDCS) is a noninvasive type of neural stimulation known for modulation of cortical excitability leading to positive effects on working memory and attention. The availability of low-cost and consumer grade tDCS devices has democratized access to such technology allowing us to explore its applicability to HCI. We review the relevant literature and identify potential avenues for exploration within the context of enhancing interactivity and use of tDCS in the context of HCI.

Permission to make digital or hard copies of part or all of this work for personal or classroom use is granted without fee provided that copies are not made or distributed for profit or commercial

advantage and that copies bear this notice and the full citation on the first page and that copies bear this notice and the full citation on the first page. Copyrights for third-party components of this work must be honored. For all other uses, contact Copyright is held by the owner/aut

, Sonoma, CA, USA $140-0 / 16 / 03$

http://dx.doi.org/10.1145/2876456.2879482

\author{
Author Keywords \\ Neurostimulation; tDCS; HCI
}

\section{ACM Classification Keywords}

H.5.2. Information interfaces and presentation.

\section{INTRODUCTION}

Transcranial direct current stimulation (tDCS) is a form of neurostimulation, which uses electrodes on the scalp to deliver constant and low electric current $(<2 \mathrm{~mA})$ to different areas of the brain [10]. This can be used to modulate cortical excitability in different brain regions [12], while remaining safe to the users. tDCS has been shown to increase cognitive performance on a variety of tasks, such as language and mathematical ability [7], attention span [8], problem solving [11], memory [3], and coordination [1], as well as treating mental disorders (e.g. Alzheimer disease [4]).

Although tDCS usually involves expensive and clinical grade equipment (e.g. Soterix Medical $1 \times 1$ tDCS Stimulator), recent releases of commercial and affordable devices have made tDCS available for public use (e.g foc.us and The Brain Stimulator [6]). These devices promise to bring the benefits of tDCS to the everyday life of a wide public population, and consequently enable exciting possibilities and scenarios for HCI. 


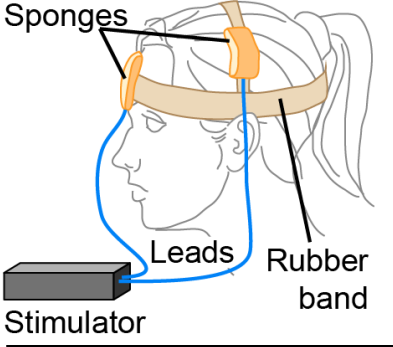

Brodmann Cortical Areas

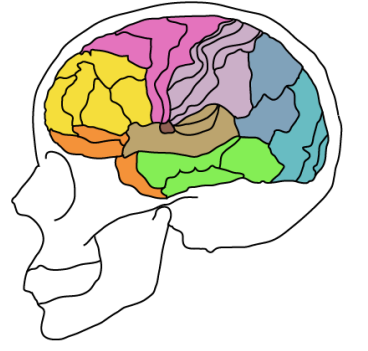

Executive functions Visual functions Emotional regualtion $=$ Sound Motor functions Memory

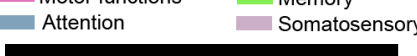

10-20 International System

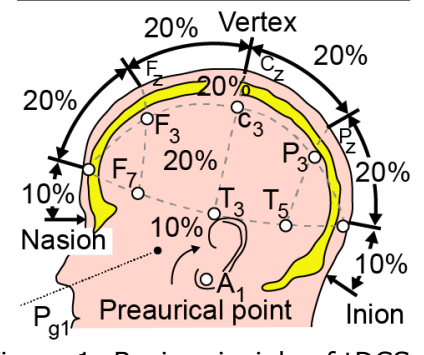

Figure 1: Basic principle of tDCS (top); anatomical functions of the human brain (middle); the 10-20 international system of electrode placements (bottom).
Medical studies give evidence that clinical tDCS devices have beneficial effect for older adults and patients with limited brain plasticity $[1,3]$. However, it is not always clear that these benefits can be translated to low-grade commodity tDCS headsets and younger users. For example, Steenbergen et al demonstrated that the first version of the commercial foc.us headset did not provide any benefit to working memory [13].

The work we present here is intended as an avenue for exploring new forms of influencing human interaction in an interactive task through the use of commodity tDCS headsets. While the ethical and moral aspect of using such a device is an important debate to have, the focus here is to highlight performance related aspects of using tDCS. In this respect the challenge lies in recognizing the capabilities of commodity tDCS devices and integrating tDCS into interactive tasks.

\section{Transcranial Direct Current Stimulation}

tDCS is a form of non-invasive neurostimulation

technique where low ampere direct current is delivered to the brain area of interest via electrodes on the scalp. This current flow increases or decreases the neuronal excitability in the area stimulated [10]. This change of neuronal excitability leads to alteration of brain function, depending on the stimulated area [10].

Traditionally, this technique is often used in clinical environments. However, with the recent introduction of low-cost commercial devices, tDCS is grabbing the attention of a wide population of users. Foc.us is one example of a commodity headset that allows users to control the location of the electrodes, voltage, current and type of stimulation. This allows a great flexibility on the tDCS technique that can be explored as well as the cognitive areas that can be stimulated. Consequently,
tDCS has a great potential to be integrated into common interactive tasks to improve various aspect of user experience in interacting with the applications.

\section{Using tDCS Stimulation for HCI}

Figure 1 (top) illustrates a basic principle of harnessing tDCS. HCI practitioners should select an area, according to the function they want to stimulate (Figure 1 middle), then place the electrodes at the correct positions in the 10-20 international system (Figure 1 bottom).

Additionally, the orientation of the electrical field, which is defined by the electrode positions and polarity, must be taken into consideration. There are usually two electrodes in tDCS: the positively charged electrode (anode) is placed at the stimulation site (region-ofinterest); the negative electrode (cathode) is usually placed on the opposite side of the body (e.g. the upper arm). Figure 2 provides some guidance on some usual electrode arrangements.

\section{Models for Integrating tDCS in HCI}

Although tDCS has been extensively researched in terms of its psychological effects, there is no research identifying the time required for it to take effect. Most studies $[3,9]$ used 5 minutes stimulation before performing the experimental task, but there is no study demonstrating the benefits of prior stimulation.

This is a key factor from an HCI perspective, as it would determine how it could be integrated with our daily tasks in a useful manner (e.g. both to improve the task at hand and for cognitive training). For instance, real time effects would enable very interesting applications for gaming (e.g. to emulate the effect of adrenaline when an enemy jumps forward shooting at us, or to avoid a crash in a racing game). 


\begin{tabular}{|l|l|l|}
\hline Montage & Effect & Reference \\
\hline F3 & $\begin{array}{l}\text { Working } \\
\text { memory }\end{array}$ & {$[3]$} \\
\hline CSA & Attention & {$[8]$} \\
\hline F3 & [SA & [2] \\
\hline Oz & $\begin{array}{c}\text { Visual } \\
\text { perception }\end{array}$ & [5] \\
\hline C2 & $\begin{array}{c}\text { Multisensory } \\
\text { perception }\end{array}$ & [5] \\
\hline CDM & Anode \\
\hline
\end{tabular}

CSA $=$ contralateral supraorbital area CSD=contralateral deltoid muscle

Figure 2: Summary of previous works on tDCS.

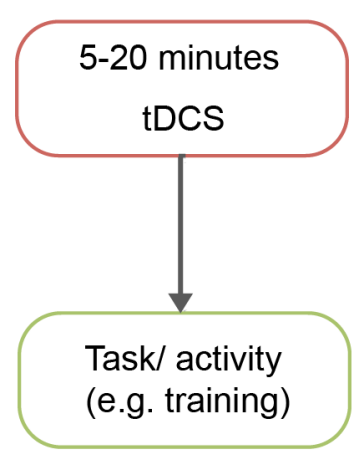

Figure 3: Open-loop model.
If the period that can produce significant effect is between 1 and 5 minutes, it can be used in quasi-realtime situations. This would be good for many scenarios we mentioned such as increasing attention when we approach a crossing where we need to take a complex turning (GPS), or looking for friends in cinema lobby. Under this $\mathrm{HCI}$ oriented perspective, we propose two models to integrate tDCS into an interactive system, which automatically and adaptively uses tDCS based on the interactive task that is being performed by the user.

\section{Open-loop integration model}

This model is designed for enhancing working memory and cognitive skills in tasks. tDCS is stimulated in a specific manner 5 to 20 mins before an activity (as in Figure 3). In this model, the anodal electrode is at F3 and cathodal electrode is at contralateral supraorbital area.

This model is beneficial to scenarios where tDCS can be applied before tasks such as gaming and training. For modern game (especially 3D game), it may take several minutes before players can actually start playing (e.g. because of loading time, introduction, network establishment, etc.). tDCS can take advantage of this period. Additionally, portable tDCS devices can be integrated into the gaming headset, which is usually wore by players. This can produce a better performance and experience in game (e.g. more concentrated and effective game). This model can be used in most HCI applications which need user to focus on the interactive procedure.

An existing example is the US Air Force Research Lab's experiment, made public in early 2014. They tried tDCS to keep soldiers awake and alert with electric shocks. The results showed that the electro-stimulation was more effecient than the use of caffeine. Although it is still at an experimental stage, the initiative proves that hijacking the brain for the end of military effectiveness will be used to justify whatever scientific means.

Closed-loop integration model

In this model, we have a task/ activity monitoring system which dynamically triggers tDCS stimulation based on current state of users. The closed-loop integration model is illustrated in Figure 4.

For example, when pupils are learning language or mathematics, if a decline in cognitive functions is detected, tDCS can be triggered to encounter this. This model can take advantage of real-time detection of user affective states using physiological sensing methods (such as EEG, GSR, fNIR). Another example is using pupil dilatation in eye tracking to detect lack of attention - cognitive decline. Constant switching between multiple windows in a desktop application could be an indication of a need for larger working memory. In these HCI scenarios, close-loop integration model with dynamic detection and adaptive trigger tDCS could typically improve the final outcome.

Another example is pilot training using unmanned attack drones. It is a very difficult and exhausting task because it needs high cognitive demands in detecting targets in complex radar images. The is considered one of the biggest bottlenecks in deploying these new, deadly weapons. The training time can be cut down significantly by delivering a mild electrical current to improve the pilot's cognitive loads and attention during training on video simulators.

\section{CONCLUSION}

In this paper we summarized useful knowledge for $\mathrm{HCI}$ practitioners to explore interactive neural stimulation. 


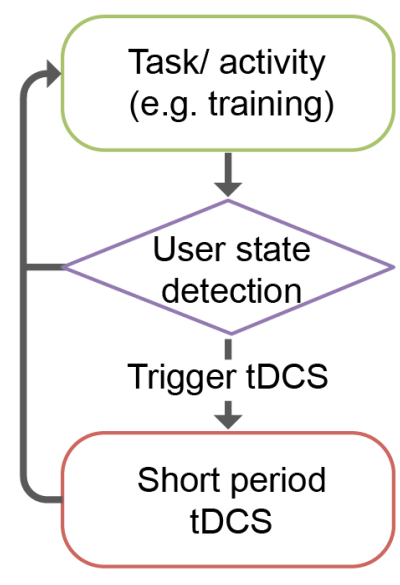

Figure 4: Closed-loop model.
We proposed two models of how to integrate tDCS into an interactive system: the open-loop model and the closed-loop model. We suggested various scenarios and $\mathrm{HCI}$ applications that can be harnessrd using the proposed two models (especially in gaming, learning and training). Future researches can continue explore in quantitative ways of how these proposed models could be harnessed in different $\mathrm{HCI}$ context (e.g. the lasting duration of the stimulation, user comfort in using tDCS device as a daily wearable device, etc.).

\section{Acknowledgements}

We thank Matt Sutton for helping with illustration. Dr. Bo Wan thanks China Scholarship Council for funding his visit to the UK to do this research.

\section{References}

1. Antal A., Nitsche M.A., Kruse W., Kincses T.Z., Hoffmann K.P., Paulus W., 2004, Direct Current Stimulation over V5 Enhances Visuomotor

Coordination by Improving Motion Perception in Humans, Journal of Cognitive Neuroscience, 16, 4: 521-527.

2. Antal A., Kincses T.Z., Nitsche M.A., Paulus W., 2003, Modulation of moving phosphine thresholds by transcranial direct current stimulation of V1 in human. Neuropsychologia, 41: 1802-1807.

3. Boggio P.S., Ferrucci R., Rigonatti S.P., Covre P., Nitsche M., Pascual-Leone A., Fregni F., 2006. Effects of transcranial direct current stimulation on working memory in patients with Parkinson's disease. Journal of the Neurological Sciences, 249: 31-38.

4. Boggio P.S., Khoury L.P., Martins D.C., Martins O.E., de Macedo E.C., Fregni F., 2009, Temporal cortex direct current stimulation enhances performance on a visual recognition memory task in Alzheimer disease, Journal of Neurol Neurosurg Psychiatry, 80, 4: 444-447.
5. Bolognini N., Olgiati E., Rossetti A., Maravita A., 2010, Enhancing multisensory spatial orienting by brain polarization of the parietal cortex. European Journal of Neuroscience, 31: 1800-1806.

6. Dubljević V., Saigle V., Racine E.,2014, The Rising Tide of tDCS in the Media and Academic Literature, Neuron, 82, 4: 731-736.

7. Flöel A., Rösser N., Michka O., Knecht S., Breitenstein C., 2008, Noninvasive Brain Stimulation Improves Language Learning, Journal of Cognitive Neuroscience, 20, 8: 1415-1422.

8. Kang E.K., Baek M.J., Kim S., Paik N.J., 2009, Noninvasive cortical stimulation improves post-stroke attention decline, Restorative Neurology and Neuroscience, 27, 6: 647-652.

9. Martin D.M., Liu R., Alonzo A., Green M., Loo C.K., 2014, Use of transcranial direct current stimulation (tDCS) to enhance cognitive training: effect of timing of stimulation, Experimental Brain Research, 232, 10: 3345-3351.

10. Medeiros L.F., de Souza I.C., Vidor L.P., de Souza A., Deitos A., Volz M.S., Fregni F., Caumo W., Torres I.L.,2012, Neurobiological Effects of Transcranial Direct Current Stimulation: A Review , Front Psychiatry, 3, 110: 1-11.

11. Metuki N., Sela T., Lavidor M., 2012, Enhancing cognitive control components of insight problems solving by anodal tDCS of the left dorsolateral prefrontal cortex, Brain Stimulation, 5, 2: 110-115.

12. Miranda P.C., Mekonnen A., Salvador R., Ruffini G. 2013, The electric field in the cortex during transcranial current stimulation, NeuroImage, 70 , 15: 48-58.

13. Steenbergen L., Sellaro R., Hommel B., Lindenberger U., Kühn S., Colzato L.S. , 2015, "Unfocus" on foc.us: commercial tDCS headset impairs working memory, Experimental Brain Research, 2015: 1-7. 